Kairos. Journal of Philosophy \& Science 22, 2019

Center for the Philosophy of Sciences of Lisbon University

\title{
Categorical Interpretation of Modal Structures under Bisimulation
}

Nino Guallart

Universidad de Sevilla

nguallart@us.es

DOI 10.2478/kjps-2019-0008

Abstract In this work we summarise the concept of bisimulation, widely used both in computational sciences and in modal logic, that characterises modal structures with the same behaviour in terms of accessibility relations. Then, we offer a sketch of categorical interpretation of bisimulation between modal structures, which comprise both the structure and the valuation from a propositional language.

Keywords: logic, modal logic, LTS, category theory, model theory.

\section{Introduction}

Bisimulation is one of the most important relations between relational structures. Informally, it can be said that bisimilar structures behave the same way in terms of accessibility. It is a relationship weaker than isomorphism, although it is the strongest behavioural relationship, which is very useful in the field of verification of formal systems, both logical and computational. Introduced in (Park, 1981) in the field of computing sciences, it has been a fruitful tool in the study of modal logic structures. 
As we will see later, the main property that characterise modal logics is bisimulation, since bisimilar models satisfy the same modal formulas. In this work we made a brief overview of relational structures and models and then we introduce the main theorems that define the bisimulation relation. Later, we will study in categorical terms the relationship of bisimulation, sketching some ideas about this topic.

Category theory offers an interesting insigth to study the relationship between different algebraic structures. In this work we propose the basics of a study of the relationship between modal logic and bisimilar models by using category theory. We can easily use categories to sketch the relationship between the category of the formulas of a modal logical language and the category of the models that satisfy them.

The paper is organized as follows. After this introduction, main notions are introduced in the second section. In the third one some theorems are studied. Then, in fourth section, categories and bisimulation are related. A short last section is devoted to conclusions, where several lines to extend categorical approaches to modal systems are pointed out.

\section{Main Concepts and Theorems}

\subsection{Relational Structures}

Relational structures are often used in semantics of formal systems, since they are able to capture the relationships and transformations between discrete elements. They can be represented as as (pseudomulti) digraphs, in which states are the nodes of the graph, and relations are the edges (Cfr. (Gerbrandy, 1999)). Here we have a basic structure over we can define now more enriched systems.

Relational structures. A relational structure is an ordered pair $\langle S, R\rangle$, where

- $\mathrm{S}$ is a set of elements or states.

- $\mathrm{R}$ is a binary relation in $\mathrm{S} x \mathrm{~S}$.

Kripke frames and labelled transition systems (LTS) are two examples of relational structures. A Kripke frame is a simple model of a relational structure, in which we consider the binary relation $\mathrm{R}$ as an accessibility relation from one state to another one. 
Definition. Kripke frame. A Kripke frame is a pair $\langle\mathrm{W}, \mathrm{R}\rangle$, where - W is a non-empty set of worlds or states.

- $\mathrm{R}$ is an accessibility relationship between states, formally defined as a binary relation in SxS.

If we consider several accessibility relations, we have a multimodal Kripke frame.

Definition. Multimodal Kripke frame. A multimodal Kripke frame is a structure $<W, A, R>$ composed by:

- A non-empty set $\mathrm{W}$ of worlds.

- A non-empty set of accessibility relationships A.

- An accessibility relation $R$ defined in WxAxW.

An alternative definition is the following: a multimodal Kripke frame is a structure $<W,\left\{R_{1}, \ldots, R_{n}\right\}_{i=1, \ldots, n}>$ composed by:

- A non-empty set W of states or worlds.

- A set of accessibility relations $R_{i}, i=1, \ldots, n$, such that every $R_{i}$ is a subset of WxW.

Labelled transition systems describe the behaviour of discrete systems, in which each world is a possible state of the system and the accessibility relations are actions or transitions between them. They have the same structure as multimodal Kripke frames.

Definition. Labelled transition system. A LTS is a relational structure $<\mathrm{W}, \mathrm{A}, \mathrm{T}>$ made up of several states (that can be understood as situations or possible worlds) and a set of arrows between them (understandable as an accessibility relationship). We have:

- A non-empty, finite set W of states or worlds.

- A non-empty, finite set A of accessibility relations, which are binary relations over the states.

- A transition relationship T, subset of $\mathrm{W} \times \mathrm{A} \times \mathrm{W}$.

LTS are widely used in computation, as graphical representation of operational semantics of programs (Keller, 1976). They are generalisations of finite automata and trees, and they are fundamental tools in concurrency theory. In this case, the stress is put in the different processes between the states instead of the states themselves, since the 
main elements of LTS are accessibility relations, instead of states. These processes are seen as transition relations between states. Thus, a LTS can be seen as a finite automaton in a graph.

In any case, if we have several accessibility relations, we write $\mathrm{w}(\mathrm{a}) \rightarrow \mathrm{u}$ to specify that there is a transition from $w$ to $u$ given by the relation $a$, instead of $\mathrm{T}(\mathrm{w}, \mathrm{a}, \mathrm{u})$ or $\mathrm{R}_{\mathrm{a}}(\mathrm{w}, \mathrm{u})$, and just $\mathrm{w} \rightarrow \mathrm{u}$ if we have only one accessibility relation.

\subsection{Models}

Now we consider a propositional logic language. If a certain proposition $p$ is true in a state $w$ in a model $M$, we say that state $w$ satisfies $p$, in symbols $M, w \vDash p$. We can consider the set of all states or worlds that satisfy a certain proposition $\mathrm{p}, \mathrm{v}(\mathrm{p})$.

Models and frames. A model is obtained when we add to a frame a valuation function $v$ : $L \rightarrow \operatorname{Pow}(W)$ from the set of atomic formulas to the power set of worlds, which contains all the subsets of the set of worlds W. Again, we can consider just one accessibility relation or several ones. From the valuation of atomic formulas, we can obtain the valuation of compound well formed formulas ("wff" from now on) via induction, and the valuation of modal formulas as we will explain soon.

Example. Modal logic over Kripke models. We have the following language:

$\varphi, \psi::=\mathrm{p}|\varphi \& \psi| \varphi \vee \psi|\varphi \rightarrow \psi| \mathrm{T}|\mathrm{F}|<>\varphi \mid[] \varphi$

We have set of states that may satisfy each atomic wff. Being $T$ and $F$ the tautology and the falsity, we recursively define the satisfaction of all wff's:

$\cdot M, w \vDash T$ for every $w$ in $M$.

- $M, w / F F$ for every $w$ in $M$ which means that no state satisfies $F$.

$\cdot M, w \vDash \varphi$ if and only if ("iff" from now on) $w$ belongs to $v(\varphi)$.

- $M, W \vDash \neg \varphi$ iff $s$ does not belong to $v(\varphi)$.

$\cdot M, w \vDash \varphi \& \psi$ iff $M, w \vDash \varphi$ and $M, w \vDash \psi$.

$\cdot M, w \vDash \varphi \vee \psi$ iff $M, w \vDash \varphi$ or $M, w \vDash \psi$.

- $M, w \vDash \varphi \rightarrow \psi$ iff $M, w$ does not satisfy $\varphi$ or $M, w \vDash \psi$. 
$\cdot M, w \vDash<a>\varphi$ iff there is $a w$ in $M$ and $a$ in $A$ such that $w(a) \rightarrow u$ and $M, u \vDash \varphi$.

- $M, w \vDash[a] \varphi$ iff for any $w$ in $M$ and $a$ in $A, w(a) \rightarrow u$ implies that $M, u \vDash \varphi$.

We can consider this a restricted case of the following example, which considers an arbitrary number of modalities.

Example. Multimodal Kripke models. We have the following language:

$\varphi::=\mathrm{p}|\varphi \& \varphi| \varphi \vee \varphi|\varphi \rightarrow \varphi| \mathrm{T}|\mathrm{F}|<\mathrm{a}>\varphi \mid[\mathrm{a}] \varphi$

Now we define the following interpretation of formulas over a multimodal Kripke frame $\mathrm{M}$, considering a set $\mathrm{A}$ of accessibility relations or modalities, being ' $a$ ' any of them. The semantics is identical to the previous one. The only difference are the definitions of the modal operators, which are as follows:

- $M, w \vDash<a>\varphi$ iff there is $a w$ in $M$ and $a$ in $A$ such that $w(a) \rightarrow u$ and $M, u \vDash \varphi$.

$\cdot M, w \vDash[a] \varphi$ iff for any $w$ in $M$ and $a$ in $A$, if $w(a) \rightarrow u$ then $M, u \vDash \varphi$.

An interesting example of a simple but powerful propositional system is Hennessy-Milner logic, used to represent transitions between states in a LTS.

Example. Hennessy-Milner logic. Hennessy-Milner logic, introduced in (Hennessy \& Milner, 1980), is a system of dynamic logic, summarized as follows:

$\varphi::=\mathrm{T}|\mathrm{F}| \varphi \& \varphi|\varphi \vee \varphi|[\mathrm{a}] \varphi \mid<\mathrm{a}>\varphi$

In this system, we have a set A of actions, where ' $a$ ' is any action. The interpretation of the wff is as follows:

$\cdot M, w \vDash T$ for every $w$ in $M$.

$\cdot M, w / F F$ for every $w$ in $M$.

$\cdot M, w \vDash \varphi \& \psi$ iff $M, w \vDash \varphi$ and $M, w \vDash \psi$.

- $M, w \vDash \varphi \vee \psi$ iff $M, w \vDash \varphi$ or $M, w \vDash \psi$.

- $M, w \vDash<a>\varphi \& \psi$ iff there is a state $u$ such that $w(a) \rightarrow u$ and $M, u \vDash \varphi$.

- $M, w \vDash[a] \varphi$ iff for every state $w$, if $w(a) \rightarrow u$ then $M, u \vDash \varphi$.

In this case, what interests us are the actions or transitions between states. Thus, if a certain state $w$ satisfies $<a>T$, it means that it is possi- 
ble to perform action a from $\mathrm{w}$, and $\langle\mathrm{b}\rangle\langle\mathrm{a}>\mathrm{T}$ means that it is possible to perform $b$ after performing $a,<a>T v<b>T$ that it is possible to perform $a$ or to perform $b$, and so on.

Note that, if we enrich this language with atomic formulas and we add the definition of $\neg \varphi$, we have the previous multimodal propositional language.

Example. A LTS with two possible actions, tick and tock is shown in the next picture. Each accessibility relation must be of one of these two types.

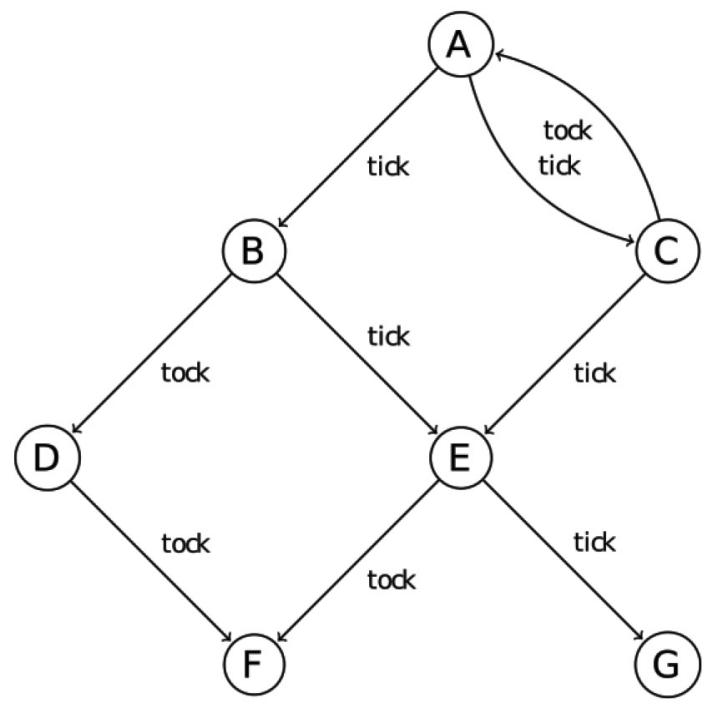

We can see that the following relations of satisfiability are satisfied:

$\mathrm{M}, \mathrm{A} \vDash<$ tick $>\mathrm{T} v<$ tock $>\mathrm{T}$

$M, B \vDash<$ tick $>T \&<$ tock $>T$

$M, D \vDash<$ tick $>F$

$\mathrm{M}, \mathrm{A} \vDash<$ tick $>(<\mathrm{tick}>\mathrm{T}$ v\& $<$ tock $>\mathrm{T})$

$\mathrm{M}, \mathrm{C} \vDash<$ tock $>$ [tick] T

For example, $D \vDash<$ tick $>\mathrm{F}$ holds because it is possible to perform action 'tick' in state $D$, more precisely from state $D$ to state $F$. $B \vDash<$ tick $>T$ $\&<$ tock $>T$ because it is the conjunction of $<$ tick $>T$ and $<$ tock $>T$, and it 
is possible to perform these two actions in state B. [tock] T means that in a given state, all transitions are given by action 'tock'. $\mathrm{C} \vDash<$ tock $>$ [tick] $\mathrm{T}$ means that it is possible to perform action 'tock' after every action 'tick' in C.

\subsection{Bisimulation in Relational Structures}

Having introduced different types of relational structures, now we can think of relations and transformations between theM, such as equivalence relations or different kinds of morphisms.

The latter can be conceived as transformations between structures, that allow to simplify or to expand them. It is interesting to explore whether the properties of the structures change or remain if we transform the original structure. For example, we might simplify or expand a structure in such a way that some of their properties remain. In this case, since these two structures share a common property, we may establish a kind of relational equivalence between them. This is the case of bisimulation between structures, in which behavioural properties of the accessibility relations hold, although the internal structure of each structure may be different. By "behavioural" properties we mean that we can perform over two bisimilar states exactly the same actions given by a wff in Hennessy-Milner logic, arriving into bisimilar states. Generalizing this definitions, two bisimilar worlds satisfy the same modal wff's in the corresponding modal language.

In order to explain bisimulation, we will introduce the concept of simulation and bisimulation, which is basically a mutual relation of simulation between two structures, and later we will explain the connection between bisimulation and modal logic.

Bisimulation between states. Two states in the same or different structures are bisimilar if they behave the same way. We can consider a Kripke frame, although bisimulation within states in a LTS would follow the same scheme, simply replacing 'accessibility relation' by 'action'. A binary relation $R$ between two states $w$ and $u$ is a simulation if there is a w' such that if $w(a) \rightarrow w^{\prime}$, then there is a u' such that $u(a) \rightarrow$ u' and w'Ru'. The union of all simulations is called similarity. A bisimulation is a simulation in both directions, from $w$ to $w$ ' and from $w$ ' to $w$. If $w$ and $\mathrm{u}$ are bisimilar, we write $\mathrm{w} \sim \mathrm{u}$. 
Bisimulation between structures and frames. If a structure $B$ is a similarity of another structure $A$, then $B$ simulates the behavioural properties of $A$, but the converse may not be the case. Indeed, simulation is a reflexive relation, but it is not symmetric, since it is possible that there is a simulation from $C_{1}$ to $C_{2}$ but not its converse. Then, a mutual simulation is called a bisimulation. In this cases, both structures behave the same way in terms of accessibility relations.

We can define more formally a bisimulation between two structures. A bisimulation $R$ between two structures $C_{1}$ and $C_{2}$ is a relation $W_{C 1} \times W_{C 2}$ between their sets of states such that that there is a simulation from the first to the second and another one to the second to the first, that is, if $w$ in $C_{1}$ and $u$ in $C_{2}$ are in the relation $R$ then these two conditions hold (forth and back, according to Van Benthem):

- (Forth): Simulation from $C_{1}$ to $C_{2}$ : if there is a w' such that if $w(a) \rightarrow w^{\prime}$, then there is an u' such that $u(a) \rightarrow u^{\prime}$ and w'Ru'.

- (Back): Simulation from $C_{2}$ to $C_{1}$ : If there is a u' such that if $u(a) \rightarrow u$ ', then there is a w' such that $w(a) \rightarrow$ w' and w'Ru'.

Two structures are bisimilar if there is a non trivial (non-empty) bisimulation between them, that is, if there are two mutual simulations between them. We can add a valuation over a given structure giving out a model, and we will see that bisimilar structures satisfy the same formulas.

Bisimulation is weaker than isomorphism, that is, two bisimilar relational structures may not be isomorphic, whereas two isomorphic structures are necessarily bisimilar. As we said before, bisimulation maintains the behavioural properties of the accessibility relations regarding a certain modal language, although the structure among the states may be different.

Example. If we consider a model given by a single state and an arrow to itself (example 1), and another one given by two states and the corresponding arrows too each other but no identity arrow (example 2), and a third one given by a linear set of states, no identity arrows (example 3 ). These three models are bisimilar, but they are not isomorphic.

Things can be even more complex, since two models can be isomorphic regarding their states and arrows, but not the set of action/agents, meaning that there is no isomorphism between the set of their actions. 
1

Picture 1

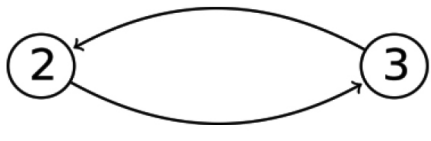

Picture 2

\section{5}

\section{6}

\section{Picture 3}

For example, let's consider the previous examples revisited with a set of two actions, tick and tock. We can see that 2 and 5 are isomorphic, but not 1 and 5, nor 3 and 6 .

As stated before, it is useful to see whether a simplification of a model holds its behavioural properties. We can see that 6 and 5 are not isomorphic, being 5 a simplification of 6 , but they are bisimilar.

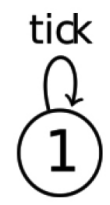

Picture 4

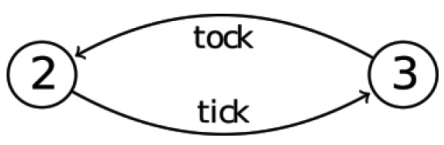

Picture 5

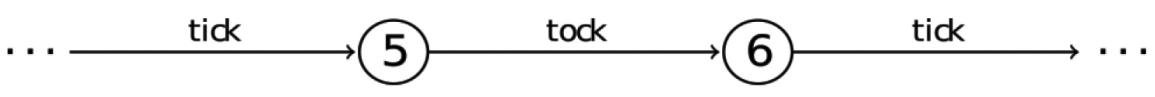

\section{Picture 6}

Simulation and bisimulation in Kripke models. If we have defined a modal propositional language $L$ and we have a Kripke frame $M$ with a set of states or worlds $\mathrm{W}$ or another kind of relational structure, we 
can add a valuation function over the states of the frame $v(p)=\{w$ in $W \mid$ $M, w \vDash p\}$. Since we have a valuation from propositions to states, we need an additional invariance clause:

- Invariance: given a proposition $\varphi, \mathrm{C}_{1}$, w is in $v(\varphi) \leftrightarrow \mathrm{C}_{2}$, $\mathrm{u}$ is in $\mathrm{v}(\varphi)$ iff wZu for each w in $C_{1}$ and each $u$ in $C_{2}$.

In this way, two states that satisfy the same wff and the previous forth and back clauses, are bisimilar in the model.

Note that we are talking about frames and models in abstract term, although it is obvious that modal logics can be interpreted in many ways, such as epistemic, temporal, deontic and other kinds of logics. Each one has its own unique features (Cfr. for example (Gerbrandy, 1999) or (Van Ditmarsch, 2007)).

\section{Theorems}

In this section we explore the basics of the relationship between modal logic and bisimilar models. In particular, three theorems that characterise modal logic via bisimulation. For a detailed explanation of the relation between bisimulation and modal logics, (Sangiorgi, 2009) or (Stirling, 2012) can be consulted.

Fundamental property of modal logic. Now we are going to explore the meaning of the assertion that states that a wff is invariant under bisimulation, and that the fundamental property of bisimulation between models is what characterise modal logic. Let's recall that a valuation function assigns a subset of states to each atomic wff, and that recursively we can define satisfaction for complex formulas and for modal wffs. The most relevant issue is the definition of valuation function for modal operators, $<\mathrm{a}>\varphi$ and $[\mathrm{a}] \varphi$. The relationship between modal logic and bisimulation is given by the next two theorems.

Theorem 1. Every wff of a language of modal propositional logic is invariant under bisimulation. That is to say, given two models $M, M^{\prime}$ and two states in them $w y$ w' (respectively), for every modal formula $\varphi$ it holds the following:

$M, w \vDash \varphi$ iff $M^{\prime}, w^{\prime} \vDash \varphi$ 
This means that the models that are bisimilar satisfy the same wff, and therefore they are indistinguishable using modal logic. Every wff that is satisfiable in a state in a model is also satisfiable in a bisimilar state.

Proof. It can be seen in (Blackburn et at, 2001, p. 67), we leave out its details to abbreviate.

Note that the aforementioned bisimilarity is between models, not just between frames. Now we see van Benthem theorem, which is key in modal correspondence theory (Cfr. Van Benthem, 1976).

Theorem 2 (van Benthem characterization theorem). Any wff invariant under bisimulation is equivalent (invariant) to a wff in modal propositional logic. That is, if two models are bisimilar, they satisfy the same modal formulas.

Proof. By definition, bisimilar states satisfy the same atomic wff. Complex formulas are also equivalent via inductive step. Modal formulas are equivalent, since by the definition of bisimulation, there are states are related to the corresponding one and satisfy the forth or back clause (See (Blackburn et at, 2001, p. 67) for a more detailed proof).

Thus, bisimulation characterise modal logic, which means that two structures cannot be distinguished in behavioural terms. Modal wff describe the behavioural properties of structures, and bisimilar structures satisfy the same formulas. In other terms, bisimulation may be seen as invariance under bisimulation. In the next part of the paper, this will be studied in categorical terms. Thus, modal logic can be seen as the fragment of first-order logic which is invariable under bisimulation (Van Benthem, 1976).

Modal equivalence. Two states, in the same or different models, are said to be modally equivalent if there are no wff with different valuation over them. The previous theorem asserts that if two wff are invariant under bisimulation, they are modally equivalent, that is, if there is a bisimulation between them, these states are modally equivalent. The converse situation is not always true: there can be the case that two states are modally equivalent but not bisimilar. Bisimulation between modal equivalent states need to verify the following theorem.

Definition. A Kripke frame is image-finite iff for every $w$ in $W$ and every accessibility relation $R$, the set $\{v \mid w R v\}$ is finite. 
The classical example of this is the following one, in which there is a structure such that there is a branch with $\mathrm{n}$ nodes for every natural number, and another structure a branch with $\mathrm{n}$ nodes for every natural number plus an infinite branch. They are bisimilar, but not modally equivalent.

Theorem 3 (Hennessy-Milner property). If two models $\mathrm{M}$ and $\mathrm{N}$ are image-finite and modally equivalent, then they are bisimilar.

Proof: See (Blackburn et at, 2001, p. 69).

Thus, bisimulation and modal equivalence are equivalent if we are restricted to the case of image-finite models (Cfr. Goranko, 2007).

\section{Categories and Bisimulation}

In this section we will apply category theory in order to study the relationship between bisimilar relational frames, such as Kripke frames or LTS. Later, we will study their relationship with the category Prop of wff of propositional logic, thus studying the corresponding models.

Firstly we recall that a category is made of the following elements:

- A class of objects, $\mathrm{Ob}(\mathrm{C})$.

- A class of arrows or morphisms between the objects; $\operatorname{Hom}(a, b)$ is the set of arrows from object a to object $b$, whereas hom(C) is the class of all morphisms of the category $C$.

Given an operation of composition of morphisms, the following rules must apply in order to consider the previous structure a category:

- Associativity: If $\mathrm{f}: \mathrm{a} \rightarrow \mathrm{b}, \mathrm{g}: \mathrm{b} \rightarrow \mathrm{c}$ and $\mathrm{h}: \mathrm{c} \rightarrow \mathrm{d}$, then $(\mathrm{fg}) \mathrm{h}=\mathrm{f}(\mathrm{gh})$.

- Identity arrow for each object $X$ in the category, $\operatorname{Id}_{\mathrm{x}}$.

Categories can be made of categories too. In this case we call the morphisms between categories functors, and the morphisms between functors, natural transformations.

\subsection{Categorical Interpretation of Frames}

In the same way that there are categories for other algebraic structures, such as Set, Grp, Top, we can consider a category LTS and another one Krp whose objects are respectively LTS's and Kripke frames, and their arrows are the different relationships between them. 
We define the category LTS in which objects are all possible LTS as objects of the category of the same name, and morphisms are the transformations between them. Analogously we can define category Krp given by all multimodal Kripke models, in which objects are all Kripke models and the arrows are the transformations between them. In the next pages, we will work on Krp, although similar operations may be induced over LTS, case for example of the Hennessy-Milner logic and its relation to LTS.

Definition. Quotient category. Given a category $C$ and an equivalence relation $\mathrm{R}$ among its morphisms, a quotient category $C / R$ is defined as a category such that its objects are those of $\mathrm{C}$ and its morphisms are the equivalence classes of $C$ in $R$. For each morphism in $C$ there is a functor $\mathrm{C} \rightarrow \mathrm{C} / \mathrm{R}$.

Now we consider all the models of Krp and the equivalence relation given by bisimulations, which is an equivalence relation. Thus we can define a quotient category from Krp given this relation.

Lemma. Bisimulation between frames is an equivalence relation.

Proof. By definition, bisimulation is symmetric. It is also reflexive, since any frame is bisimilar to itself. Transitivity can be proven by considering three frames, $W, Y$ and $Z$, such that $W$ and $Y$ are bisimilar and $Y$ and $Z$ are bisimilar. From the definition of bisimulation, it is immediate that $\mathrm{W}$ and $Z$ are bisimilar.

This allows us to define now equivalence classes over the categories based on the bisimulation equivalence relation. Since bisimulation is an equivalence relation, it determines a series of quotient categories, analogous to a quotient set but in categorical terms. Each one is made up of all frames that are bisimilar among them.

Quotient category Krp/bisim. If we consider the category Krp, we study the equivalence relation given by the bisimulation between frames, that defines the quotient category Krp/bisim. The morphisms of this category are the equivalence classes of morphisms in Krp, and the objects are the same of Krp. We can consider a category whose objects are the arrows of Krp/bisim as representatives of each equivalence class, considering as arrows of this category:

- Identity morphism.

- Possible morphisms between subcategories. 


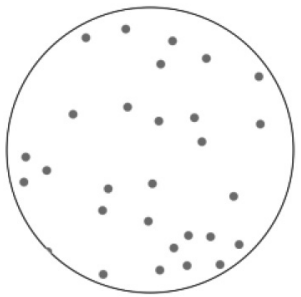

Category Krp

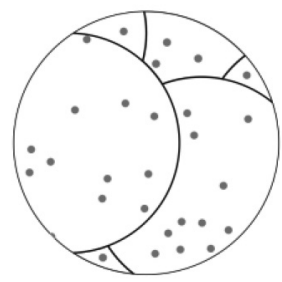

Quotient category on Krp

In the same way, we can define the category Krp/*, defined as the quotient category defined by the structural properties of the frames, for example being reflexive or transitive.

\subsection{Categorical Interpretation of Propositional Modal Languages}

In order to define valuation, we have to consider a third category, Prop, the category of all propositions in a given formal language.

Prop, ModalProp. Category Prop is defined as follows:

$\varphi::=\mathrm{F}|\mathrm{T}| \mathrm{b}|\varphi+\varphi| \varphi \times \varphi \mid \varphi \rightarrow \varphi$

$F$ and $T$ are the so-called initial and terminal objects, which can respectively be identified with the categorical interpretations of the falsity and the tautology, whereas b is an arbitrary atomic object of the category, which is the interpretation of an atomic formula. The categorical model of a propositional logic are bicartesian closed categories (biCCCs). Categorical structures are to be understood in an intuitionistic way, since in a biCCC we interpret objects as propositions and morphisms as their proofs. Let's recall that the main difference between intuitionistic and classical logic is that the first one, the principle of excluded middle and the elimination of double negation do not hold. Some classical tautologies are not intuitionistic tautologies; $p \vee \neg p$ is a classical tautologý, but not an intuitionistic one, because we need either a proof of $p$ or a proof or $\neg p$, and it may be the case that we have none of them. Since it corresponds to an intuitionistic logic system, wome additionl axioms should be added in order to consider a classical system, for example $\neg \neg \varphi \rightarrow \varphi$ for every proposition $\varphi$. If we want to consider a modal logic, we need to add the appropriate categorical equivalents of modal operators. 
Monads and comonads. Monads are endofunctors equipped with two natural transformations. Endofunctors are functors from a category onto itself, whereas natural transformations are.

For a given category $\mathrm{C}$ and a given monad $\mathrm{T}$ there are two natural transformations, $\eta: 1_{C} \rightarrow T$ (being $1 C$ the identity functor on $C$ ) and $\mu: \mathrm{T} \times \mathrm{T} \rightarrow \mathrm{T}$, which must satisfy the following coherence conditions:

$\cdot \mu * T \mu=\mu * \mu T$

$\cdot \mu \mathrm{T} \eta=\mu \eta \mathrm{T}=1_{\mathrm{T}}$

A comonad is the categorical dual of a monad. Monads and comonads are commonly used in order to categorise modal operators. <> can be understood categorically as an endofunctor from a propositional category to itself. [] is less satisfactorily equivalent to its dual. If we consider not only a single modality but a family of them, we can understand them as a family of endofunctors, each one for each modality.

Category ModalProp is defined as follows:

$\varphi::=\mathrm{F}|\mathrm{T}| \mathrm{b}|\varphi+\varphi| \varphi \times \varphi|<\mathrm{a}>\varphi|[\mathrm{a}] \varphi$

The only difference from the category Prop is the addition of $<a>$, which is interpreted as a the categorical counterpart of a modal operator, being ' $a$ ' an element of a non-empty set A of modalities, or simply <> if we are considering just one. We can consider this category as a kind of CS4-category, a bi-CCC with coproducts, a monad and a comonad, since composition of monads and comonads is reflexive and transitive (Alechina et at, 2001).

The category ModalProp/* is an quotient class, in which objects are wff that have the same logical structure, meaning that we can consider them as wff of a quantified propositional logic; in this way, $(\varphi)<a>(\varphi \rightarrow \varphi)$ is the class of all formulas that can be obtained substituting $\varphi$ by any wff, that is, $<a>(\varphi \rightarrow \varphi)$ for any $\varphi)$.

We can also consider the following HML category, which is defined as follows:

$$
\varphi::=\mathrm{F}|\mathrm{T}| \varphi+\varphi|\varphi \times \varphi| \varphi \rightarrow \varphi|<\mathrm{a}>\varphi|[\mathrm{a}] \varphi
$$

We do not have other objects other than the initial and terminal objects, and their compositions. Given a family of actions, this category is use- 
ful for modelling the composition of transitions from an initial state to another one, understanding objects as states and the corresponding functors as actions.

In both cases, it is easy to see that these categories are semantic categorical interpretations of first order logic languages, where the conjunction of two formulas is the product of them, and the disjunction equates to the categorical sum and implication to function application.

\subsection{Categorical Interpretation of Properties over Frames and Models}

This section is the final step of the study that has been established in this paper, establishing in a succint way possible lines of application of category theory to the study of modal logic. As has been said, bisimulation in modal frames and models allows to establish equivalence classes of them, and therefore quotient sets. This means that each equivalence class determines a class of frames that satisfy the same modal wffs.

We have studied this relationship in terms of categories. If we consider the categories of Kripke frames and their quotient sets regarding bisimulation, we have seen that we obtain the corresponding quotient categories over them.

The central point of the categorical study of bisimulation if the following: If we consider the category Prop, the fundamental property of bisimulation can be seen in a very simple way: since all bisimilar models satisfy the same modal wff's, the valuation function can be represented with a fuctor from the category ModalProp of modal wff's to the quotient category of bisimilar models, Krp/bisim.

It would be possible to set a functor from to ModalProp to Krp/*, establishing a relation between the propositions of the first category and the objects of the second one, that are classes of frames that hold a certain property, such as every proposition characterises a certain property of frames, for example being reflexive. Actually, what we define is a pair of adjoint functors, I: ModalProp $\rightarrow \mathrm{Krp} /{ }^{*}$ and L: Krp/* $\rightarrow \mathrm{Krp} / \mathrm{bisim}$ such as the first one is the left adjoint of the second one. The basic idea is that a certain property, expressed in modal logic, corresponds to a class of equivalent frames, and conversely, a certain class of equivalent frames corresponds to a certain modal property. 
For example, the following formulas define possible properties of frames:

- [] $\varphi \rightarrow \varphi$ defines the subcategory of reflexive frames.

- [] $\varphi \rightarrow[][] \varphi$ defines the subcategory of transitive frames.

- $\varphi \rightarrow[]<>\varphi$ defines the category of symmetric frames.

More complex subcategories are possible via all possible combinations. The subcategory of reflexive and transitive frames (S4) is nothing but the intersection of these, for example.

We consider this line of research fruitful, and we leave the development of this issue for further works.

\section{Concluding Remarks}

In this work we have done a brief introduction to relational structures and later we have studied the notion of bisimulation between them, with special emphasis on the relation between bisimulation and modal logic. In the second part of this work we have sketched an outline of a categorical interpretation of bisimulation. More precisely, we have seen that the fundamental property of modal logic can be represented in category theory in a very straightforward way. Possible future works may extend this categorical approach in different directions:

- A detailed study of the categorical interpretation of Kripke models.

- A study in categorical terms the different modal axiomatic systems, such as K, T, S4 or S5 and their corresponding properties, exploring what kind of subcategory of Krp determines each axiomatic schemata, and studying it in relation to the logical systems that each element of quotient category defines.

- The relationship between Hennessy-Milner and coalgebraic modal logics in category theory.

- A further study of the relationship between game semantics and bisimulation, since actions can be seen as moves in a dialogical game. 


\section{Bibliography}

(1) Alechina, N., Mendler, M., De Paiva, V., \& Ritter, E. (2001, September). Categorical and Kripke semantics for constructive S4 modal logic. En International Workshop on Computer Science Logic (pp. 292-307). Springer Berlin Heidelberg.

(2) Van Benthem, J. (1976). Modal correspondence theory [Ph.D. Thesis]. University of Amsterdam, Netherlands.

(3) Blackburn, P., De Rijke, M., \& Venema, Y. (2001). Modal logic, volume 53 of Cambridge tracts in theoretical computer science.

(4) Van Ditmarsch, H., van Der Hoek, W., \& Kooi, B. (2007). Dynamic epistemic logic (Vol. 337). Springer Science \& Business Media.

(5) Gerbrandy, J.D. (1999). Bisimulations on planet Kripke. ILLC Dissertation Series.

(6) Goranko, V., \& Otto, M. (2007). 5 Model theory of modal logic. Studies in Logic and Practical Reasoning, 3, 249-329.

(7) Hennessy, M., \& Milner, R. (1980). On observing nondeterminism and concurrency. Automata, Languages and Programming, 299-309.

(8) Keller, R.M. (1976). Formal verification of parallel programs. Communications of the ACM, 19(7), 371-384.

(9) Park, D. (1981). Concurrency and automata on infinite sequences. In Theoretical computer science (pp. 167-183). Springer Berlin Heidelberg.

(10) Sangiorgi, D. (2009). On the origins of bisimulation and coinduction. ACM Transactions on Programming Languages and Systems (TOPLAS), $31(4), 15$.

(11) Stirling, C. (2012). Bisimulation and logic. Sangiorgi and Rutten [24, Chapter 4], 173-196.

(12) Venema, Y. (2007). 6 Algebras and coalgebras. Studies in Logic and Practical Reasoning, 3, 331-426. 